Andreas Pangritz*

\title{
Jüdisches Leben in Deutschland als Anfrage an die innerchristliche ökumene
}

https://doi.org/10.1515/mdki-2021-0001

Zusamenfassung: Aus Anlass des Jubiläums „1700 Jahre jüdisches Leben in Deutschland“ wird erörtert, wie die „eine tatsächlich große ökumenische Frage“ (K. Barth), nämlich die Beziehungen des Christentums zum Judentum, beantwortet werden kann. Gebraucht wird ein neues Konzept von Ökumene, das über H. Meyers „Einheit in versöhnter Verschiedenheit“ hinausgeht: „Versöhnte Verschiedenheit in dialogischer Differenz", eine dialogische Weggemeinschaft unter Anerkennung bleibender Verschiedenheit.

Abstract: In the context of the jubilee " 1700 years of Jewish life in Germany", the author discusses how the "one really great ecumenical question" (K. Barth), namely the relationship between Christianity and Judaism, can be addressed. A new concept of ecumenism that goes beyond H. Meyer's vision of "unity in reconciled diversity" is necessary: a concept that understands ecumenical reconciliation as encounter across boundaries of persisting diversities without seeking to ensnare the other for one's own interests.

\section{Einleitung}

Der Verein „321-2021: 1700 Jahre jüdisches Leben in Deutschland“, der im April 2018 im Gemeindehaus der Synagoge Köln gegründet wurde, hat sich laut Homepage des Vereins zum Ziel gesetzt, „die Bedeutung der jüdischen Kultur und Geschichte für Deutschland und Europa wachzuhalten“. Er möchte „dazu beitragen, kulturelle, politische und interreligiöse Debatten innerhalb der Gesellschaft anzustoßen und deutliche Zeichen gegen den wachsenden Antisemitismus zu setzen“. Als Anlass wird die Erinnerung an ein Edikt des römischen Kaisers Konstantin vom 11. Dezember 321 genommen, in dem festgelegt wurde, „dass Juden städtische Ämter in der Kurie, der Stadtverwaltung Kölns, bekleiden dürfen und sollen“. Durch dieses Edikt werde belegt, „dass jüdische Gemeinden bereits seit der Spätantike wichtiger integrativer Be-

*Kontakt: Andreas Pangritz, E-Mail: pangritz@uni-bonn.de standteil der europäischen Kultur sind“. Deshalb könne von einer „mehr als 1700 Jahre alten jüdischen Geschichte in Deutschland und Europa“ geredet werden. Dies soll mit dem Festjahr 2021 gefeiert werden.

Zwar mag aufgrund des Mangels an historischer Überlieferung bezweifelt werden, dass von einer kontinuierlichen Präsenz jüdischen Lebens über 1700 Jahre seit der Antike im Rheinland gesprochen werden kann; erst ab dem 10. Jahrhundert werden jüdische Gemeinden insbesondere in den drei SchUM-Städten Speyer, Worms und Mainz wieder sichtbar. Das ändert aber nichts daran, dass die jahrhundertelange Präsenz jüdischen Lebens in den deutschen Territorien bislang von den christlichen Kirchen wie auch von der deutschen Gesellschaft viel zu wenig wahrgenommen und in ihrer Bedeutung für das eigene Selbstverständnis reflektiert worden ist. Diesem Mangel will die Initiative „1700 Jahre jüdisches Leben in Deutschland“" abhelfen.

Mit gutem Grund haben sich christliche Kirchen, Gruppen und Einzelpersonen in die Initiative eingebracht, um die jahrhundertelange Präsenz von Juden und jüdischen Gemeinden in Deutschland und Europa mitzufeiern. Denn tatsächlich stellt sich bei näherem Hinsehen heraus, dass die Ausbreitung des Christentums in Europa von Anfang an durch ein jüdisches Gegenüber begleitet und herausgefordert war. Dabei gestaltete sich die Begegnung von Christen und Juden durch die Jahrhunderte durchaus unterschiedlich: Freundschaftliche oder geschäftsmäßige Beziehungen wechselten mit Zeiten der Konfrontation, in denen die Christen - begünstigt durch ihre staatliche Privilegierung - zu Verfolgern der Juden wurden. Letzteres war insbesondere seit dem ersten Kreuzzug (1096) der Fall, als die Kreuzfahrer über jüdische Gemeinden im Rheinland herfielen, um den im Kreuzestod Jesu angeblich vollbrachten Gottesmord an den als Täter verleumdeten Juden $\mathrm{zu}$ rächen.

Vor diesem Hintergrund ist die traditionelle Rede von einem „christlichen Abendland“ schon immer fragwürdig gewesen, da sie die jüdische Präsenz ignoriert. Aber auch in der heute verbreiteten Rede vom ,jüdisch-christlichen“ Erbe Europas kommt ein verzerrtes Geschichtsbild zum Ausdruck, da hier der Aspekt des Konfliktes und der Konfrontation in der Begegnung von Christen und Juden durch einen harmonisierenden Bindestrich nivelliert wird. Zum einen wird damit ausgeblendet, dass neben 
dem Judentum und dem Christentum auch der Islam seit Jahrhunderten zum europäischen Erbe zählt. Zum andern wird damit ausgeblendet, dass durch den europäischen Antisemitismus, der trotz anderslautender Behauptungen nicht zuletzt auf christliche Wurzeln zurückgeführt werden muss, das christliche Erbe Europas selbst ad absurdum geführt worden ist: Die Transformation traditioneller christlicher Judenfeindschaft in die mörderische Ideologie des Nationalsozialismus, die mit der Schoa in dem Versuch mündete, jüdisches Leben in Europa auszulöschen, hat die Problematik der Ideologie vom ,christlichen Abendland“ in einer zuvor unvorstellbaren Weise ans Licht gebracht.

Umso erstaunlicher ist die Tatsache, dass heute wieder eine jüdische Zuwanderung nach Europa und nicht zuletzt nach Deutschland beobachtet werden kann, so dass mit gutem Grund 1700 Jahre jüdischer Präsenz in Europa gefeiert werden können. $\mathrm{Zu}$ bedenken ist jedoch, dass die Mitgliedszahlen jüdischer Gemeinden nach der Statistik des Zentralrats der Juden in Deutschland seit 2005 wieder rückläufig sind - Ende 2019 vereinten die jüdischen Gemeinden knapp 95.000 Mitglieder -, so dass die Zukunft der jüdischen Gemeinschaft hierzulande keinesfalls als gesichert gelten kann.

Zugleich bedeutet jüdisches Leben in Deutschland heute eine Herausforderung für die innerchristliche Ökumene, die bis jetzt noch kaum wahrgenommen worden ist. Im Selbstverständnis der ökumenischen Bewegung, die unter dem Motto der "versöhnten Verschiedenheit“ freundschaftliche Beziehungen zwischen den christlichen Konfessionen fördern will, spielt die Präsenz von Juden bis heute kaum eine Rolle. Die schon bald nach der Schoa begründeten Gesellschaften für christlich-jüdische Zusammenarbeit, die sich nicht zuletzt die Bekämpfung des hierzulande nach wie vor grassierenden Antisemitismus zur Aufgabe gemacht haben, werden etwa von der Arbeitsgemeinschaft Christlicher Kirchen in Deutschland oder auch von den einzelnen Kirchen kaum als ökumenisch tätige Partner wahrgenommen. Wo sich christliche Kirchen in ihrem Verhältnis zum Judentum zur Umkehr aufgerufen sehen wie etwa die Römisch-katholische Kirche mit der Erklärung Nostra aetate (Über das Verhältnis der Kirche zu den nichtchristlichen Religionen, 1965) und die Evangelische Kirche im Rheinland mit ihrem Beschluss Zur Erneuerung des Verhältnisses von Christen und Juden (1980), da geschieht das in der Regel unabhängig voneinander. Eine erfreuliche Ausnahme stellt die Charta Oecumenica der Konferenz Europäischer Kirchen und des Rats der Europäischen Bischofskonferenzen aus dem April 2001 dar, die im Kapitel über „Unsere gemeinsame Verantwortung für Europa“ neben anderen Aspekten auch die
Vertiefung der „Gemeinschaft mit dem Judentum“ als Ziel nennt. $^{1}$

Die folgenden Überlegungen möchten deutlich machen, dass die Tatsache jüdischen Lebens in Deutschland nicht nur aus soziologischen oder sozialethischen, sondern auch aus theologischen Gründen als eine Anfrage an das ökumenische Selbstverständnis christlicher Kirchen verstanden werden sollte, die zu einem neuen Verständnis von Ökumene führen könnte. Ich will dabei in drei Schritten vorgehen: Zunächst werde ich anhand der in der innerchristlichen Ökumene verbreiteten Redeweise von der „versöhnten Verschiedenheit“ auf Probleme des Selbstverständnisses der ökumenischen Bewegung hinweisen; danach werde ich über die von Karl Barth geprägte These nachdenken, wonach „unsere Beziehung zum Judentum“ die „eine tatsächlich große ökumenische Frage" sei; und abschließend werde ich fragen, welche Bedeutung Barths Perspektive für einen veränderten Begriff von Ökumene haben könnte.

\section{2 Ökumene als „Einheit in versöhnter Verschiedenheit"}

Die Kurzformel „Einheit in versöhnter Verschiedenheit“ ist vor bald fünfzig Jahren von dem lutherischen Theologen Harding Meyer geprägt worden, der lange Jahre am Institut für Ökumenische Forschung in Straßburg tätig war. ${ }^{2}$ Die Formel wird seither gerne zur Beschreibung des Ziels der ökumenischen Bewegung verwendet, wobei insbesondere an die Beziehung zwischen Römisch-katholischer und Lutherischen Kirchen gedacht wird. Sie ist jedoch so allgemein gehalten, dass sie auch auf andere Spannungsverhältnisse innerhalb der Ökumene angewandt werden kann. Zugleich ist sie so flexibel, dass sie inhaltlich durchaus verschieden verstanden werden kann. So macht es z. B. keinen geringen Unterschied, ob die Betonung eher

1 Vgl. Charta Oecumenica. Leitlinien für die wachsende Zusammenarbeit unter den Kirchen Europas, Genf / St. Gallen, April 2001, §10: „Wir verpflichten uns, allen Formen von Antisemitismus und Antijudaismus in Kirche und Gesellschaft entgegenzutreten“ und ,auf allen Ebenen den Dialog mit unseren jüdischen Geschwistern zu suchen und zu intensivieren“. Wortlaut unter www.oekumene-ack.de/fileadmin/ user_upload/Charta_Oecumenica/Charta_Oecumenica.pdf.

2 Vgl. Harding Meyer, „Versöhnte Verschiedenheit“ - Korrekturen am Konzept der „konziliaren Gemeinschaft“, in: Lutherische Monatshefte 14 (1975), 675-679. Vgl. ders., „Einheit in versöhnter Verschiedenheit“ - „konziliare Gemeinschaft“ - „organische Union“. Gemeinsamkeit und Differenz gegenwärtig diskutierter Einheitskonzeptionen, in: Ökumenische Rundschau 27 (1978), 377-400. 
auf die Einheit oder auf die Verschiedenheit gelegt wird. Im einen Fall läuft die Zielvorstellung eher auf eine Einheit der Kirche durch Überwindung der Differenzen hinaus, im anderen eher auf eine Zusammenarbeit bleibend verschiedener kirchlicher Gemeinschaften. Versöhnung würde im einen Fall eher auf Vereinigung hinauslaufen, im anderen eher auf Verständigung.

Auf der dritten Vollversammlung des Ökumenischen Rates der Kirchen in Neu-Delhi (1961) ist eine „Einheitsformel“ als Zielvorstellung der ökumenischen Bewegung verabschiedet worden, in der die folgenden Elemente als konstitutiv für die kirchliche Einheit genannt werden: „Bekenntnis des einen apostolischen Glaubens, Verkündigung des einen Evangeliums, Gemeinsamkeit in der einen Taufe, in der einen Eucharistie, im geistlichen Amt, im Gebet und im Leben“. Diese Elemente der Einheit sollten eine ,völlig verpflichtete Gemeinschaft“ konstituieren, die „sich in Zeugnis und Dienst an alle wendet“. ${ }^{3}$ Für diese in Neu-Delhi formulierte Zielvorstellung der Einheit von Kirchen sind in der Folgezeit dann auch Formeln wie „konziliare Gemeinschaft“ und „organische Union“ verwendet worden. ${ }^{4}$ Der Ausdruck „organische Union“ wurde dann auf der fünften Vollversammlung des Ökumenischen Rats der Kirchen in Nairobi (1975) näher umschrieben als eine „transkonfessionelle Gestalt kirchlicher Einheit“, in der die „konfessionellen Besonderheiten und Traditionen durch Verschmelzung der Konfessionskirchen zu ,einer einzigen Körperschaft"“ aufgehoben werden. ${ }^{5}$

In einer Parallelbewegung hat die Römisch-katholische Kirche, die sich selbst als die eine ökumenische Kirche versteht, auf dem Zweiten Vatikanischen Konzil am 21. November 1964 mit Unitatis Redintegratio ein Dekret über den „Ökumenismus“ verabschiedet, in dem die Zielvorstellung einer Wiederherstellung kirchlicher Einheit aus römischer Sicht formuliert wird. Die ökumenische Bewegung wird hier als eine „sich von Tag zu Tag ausbreitende Bewegung zur Wiederherstellung der Einheit aller Christen“ gewürdigt, die „unter unsern getrennten Brüdern [...] unter der Einwirkung des Heiligen Geistes“ entstanden sei (1). Unter Hinweis auf das hohepriesterli-

3 Neu-Delhi 1961. Dokumentarbericht über die Dritte Vollversammlung des Ökumenischen Rates der Kirchen, hg. v. Willem A. Visser 't Hooft, Stuttgart 1962, 130; zit. bei Harding Meyer, „Einheit in versöhnter Verschiedenheit“. Hintergrund, Entstehung und Bedeutung des Gedankens, in: ders., Versöhnte Verschiedenheit. Aufsätze zur ökumenischen Theologie, Bd. I, Frankfurt a.M. / Paderborn 1998, 101-119, hier: 103 (Hervorhebungen AP).

4 Vgl. Meyer, „Einheit“ (s. Anm. 3), 110.

5 Bericht aus Nairobi 1975. Offizieller Bericht der 5. Vollversammlung des ÖRK, Frankfurt a.M. 1976; zit. bei Meyer, „Einheit“ (s. Anm. 3), 110 . che Gebet Jesu, „dass alle eins seien“ (Joh 17,21), auf das von ihm gestiftete „wunderbare Sakrament der Eucharistie [...], durch das die Einheit der Kirche bezeichnet und bewirkt" werde, und auf das Petrusamt, das dazu diene, die Schafe „in vollkommener Einheit“ zu weiden (2), wird die in der katholischen Kirche bereits verwirklichte Kircheneinheit umschrieben. Es wird aber auch eingeräumt, dass in „dieser einen und einzigen Kirche Gottes [...] schon von den ersten Zeiten an Spaltungen entstanden“ seien, die „zur Trennung recht großer Gemeinschaften von der vollen Gemeinschaft der katholischen Kirche“ geführt hätten. Die Glieder dieser „getrennten Kirchen und Gemeinschaften" sollten von der katholischen Kirche „als Brüder im Herrn anerkannt“ werden, die „in einer gewissen, wenn auch nicht vollkommenen Gemeinschaft mit der katholischen Kirche“ ständen (3). Ziel der „ökumenischen Betätigung“ ist hier die „Wiederversöhnung aller Christen in der Einheit der einen und einzigen Kirche Christi“ (24).

Gemeinsam ist den zitierten Erklärungen, dass hier eine sichtbare institutionelle Einheit der Kirche als Ziel der ökumenischen Bewegung propagiert wird, die zugleich als die Wiederherstellung einer fiktiven ursprünglichen Einheit gesehen wird. Zwar wird immer wieder betont, dass Einheit keinesfalls „Uniformität“ bedeuten solle. „Einheit und Vielgestaltigkeit, Gemeinschaft und Verschiedenheit" schlössen einander nicht aus, sondern gehörten zusammen. „Einheit der Kirche“ wurde also durchaus als „Einheit in Verschiedenheit“ gedacht. ${ }^{6}$ Dabei war jedoch zunächst nur an kulturell bedingte Differenzen wie etwa die der Sprache oder der liturgischen Traditionen gedacht, nicht an eine Koexistenz bleibend verschiedener Konfessionen.

Das von Harding Meyer entwickelte Konzept der „Einheit in versöhnter Verschiedenheit“ setzt gegenüber den bisher genannten Einheitskonzepten durchaus neue Akzente: Hatten frühere Einheitsvorstellungen darauf insistiert, dass das Ziel der Einheit letztlich auf eine Überwindung der konfessionellen Differenzen hinauslaufe, so sollen in der Idee der „versöhnten Verschiedenheit“ die bleibend verschiedenen Konfessionen gerade für eine ökumenische Versöhnung fruchtbar gemacht werden. Die konfessionellen Weltbünde der Lutheraner, Reformierten, Methodisten usw. werden jetzt nicht mehr als durch die ökumenische Bewegung zu überwindendes Problem, sondern selber als Akteure der ökumenischen Bewegung

6 Meyer, „Einheit“ (s. Anm. 3), 105. 
ins Spiel gebracht. ${ }^{7}$ Neben der Gewährung von „Kirchengemeinschaft" trotz fortbestehender konfessioneller Differenzen kann auch auf die Feststellung von „Konvergenzen“ aufgrund von interkonfessionellen Dialogen hingewiesen werden, die zwar keinen vollen „Konsens“ formulieren, aber doch „trennende Gegensätze aufheben und eine Verwirklichung kirchlicher Gemeinschaft ermöglichen" sollen. ${ }^{8}$

Meyer will sowohl in der Gewährung voller „Kirchengemeinschaft“ als auch in der Herausarbeitung von „Konvergenzen“ „Parallelkonzeptionen“ zum Konzept der „Einheit in versöhnter Verschiedenheit“" verstehen. ${ }^{9}$ Die „eigentliche Pointe des Gedankens einer ,Einheit in versöhnter Verschiedenheit““ bestehe ja darin, „dass die Einheit der Kirche, die wir suchen, in sich Verschiedenheiten umfassen kann und darf, zu denen auch konfessionelle Verschiedenheiten hinzugehören“ ${ }^{10}$ Es geht um „die Möglichkeit einer Kirchengemeinschaft zwischen bekenntnisverschiedenen, und zwar bleibend bekenntnisverschiedenen Kirchen“. ${ }^{11}$

Es hat auch Kritik am Konzept der „versöhnten Verschiedenheit" gegeben: Dabei wurde insbesondere gefragt, „ob denn eine Gemeinschaft ,versöhnter Verschiedenheit' wirklich eine ,völlig verpflichtende Gemeinschaft" bilde oder nicht doch nur ein lockeres Nebeneinander von selbständigen und letztlich untereinander unabhängiger Kirchen““ ${ }^{12}$ Demgegenüber betont Meyer, dass hier „keine lockere Allianz“ gemeint sei, „aus der sich die Partner jederzeit wieder lösen können“. Es sei jedoch tatsächlich nötig, das Konzept zu „vertiefen“, indem etwa nicht nur von „versöhnter Verschiedenheit“, sondern ausdrücklich von „Einheit in versöhnter Verschiedenheit“ geredet werde, so dass „das Element der ,Versöhnung“ und der ,Einheit““ stärker inhaltlich hervortrete. Dazu gehöre dann auch „die Frage nach den Strukturen einer solchen Einheit". ${ }^{13}$

7 Vgl. Harding Meyer, „,Einheit in versöhnter Verschiedenheit‘, eine ökumenische Zielvorstellung. Hintergrund - Entstehung - Bedeutung - Anfragen“, in: ders., Versöhnte Verschiedenheit. Aufsätze zur ökumenischen Theologie, Bd. III, Frankfurt a.M. / Paderborn 2009, 17-40, hier: 23.

8 Meyer, „Einheit“ (s. Anm. 3), 107.

9 Meyer, „Einheit“ (s. Anm. 3), 107, Anm. 14.

10 Meyer, „Einheit“ (s. Anm. 3), 108.

11 Meyer, „Einheit“ (s. Anm. 3), 109.

12 Meyer, „Einheit“ (s. Anm. 3), 114.

13 Meyer, „Einheit“ (s. Anm. 3), $114 \mathrm{f}$.

\section{Die „eine tatsächlich große ökumenische Frage“}

Karl Barth hat das christlich-jüdische Verhältnis ins Zentrum seiner ökumenischen Perspektive gerückt. ${ }^{14}$ In bewusster Auseinandersetzung mit dem rassistischen Antisemitismus der Nationalsozialisten und der Deutschen Christen, insbesondere aber auch mit dem bis in die Bekennende Kirche hinein verbreiteten christlichen Antisemitismus in der Tradition Martin Luthers und Adolf Stoeckers hat Barth in seiner Kirchlichen Dogmatik betont, dass die Bibel Alten und Neuen Testamentes ein Erzeugnis des „jüdischen Geistes“ sei. ${ }^{15}$ Gegen den neuheidnischen Geist der Zeit und die Israelvergessenheit der christlichen Tradition insistiert Barth damit auf den jüdischen Wurzeln des christlichen Glaubens.

Die israeltheologische Tragweite dieser Erkenntnis wird von Barth in der Gotteslehre der Kirchlichen Dogmatik verdeutlicht. Dort entfaltet er im Rahmen der „Lehre von der Gnadenwahl“ seine sog. „Israellehre“, indem er die Erwählung der Gemeinde in ihrer Doppelgestalt als „Israel und die Kirche“ unter dem „Bogen des einen Bundes“ behandelt, der sich „über das Ganze wölbt“. ${ }^{16}$ Bezeichnenderweise stützt sich Barth für seine Auslegung der Kapitel 9-11 des Römerbriefs in diesem Zusammenhang nicht zuletzt auf die Schrift Die Judenfrage des methodistischen Theologen Ernst Ferdinand Ströter (1846-1922). ${ }^{17}$ Als ein Anhänger der Lehre von der „Allversöhnung“ hatte Ströter die antijudaistische theologische Tradition, wonach die Juden aufgrund ihrer Ablehnung der Messianität Jesu von Gott verworfen und als auserwähltes Volk durch die christliche Kirche ersetzt worden seien, in Frage gestellt. Barth meint von Ströters Schrift, dass sie ,trotz ihrer kräftigen Irrtümer“ immer noch „nützlich zu lesen“ sei. ${ }^{18}$

Dass mit der Frage nach dem Verhältnis der Kirche zu den Juden für Barth das Zentrum der Dogmatik berührt

14 Vgl. zum Folgenden ausführlicher: Andreas Pangritz, „Die ,eine große ökumenische Frage': Karl Barths Entdeckung der theologischen Bedeutung des Judentums“, in: Martin Bock u. Wolfgang Hüllstrung (Hg.), Karl Barth und der interreligiöse Dialog heute. Vorträge im Karl-Barth-Jahr 2019 an der Melanchthon-Akademie Köln, Düsseldorf 2020, 20-29.

15 Karl Barth, Die Kirchliche Dogmatik, Bd. I: Die Lehre vom Wort Gottes. Prolegomena zur Kirchlichen Dogmatik. Zweiter Teilband, Zollikon-Zürich 1938, 566.

16 Karl Barth, Die Kirchliche Dogmatik, Bd. II: Die Lehre von Gott. Zweiter Teilband, Zollikon-Zürich 1942, 213-336, hier: 220.

17 Vgl. Ernst Ferdinand Ströter, Die Judenfrage und ihre göttliche Lösung nach Römer Kapitel elf, Kassel 1903; 2. Aufl. Bremen o. J. [um 1922].

18 Barth, Die Kirchliche Dogmatik, Bd. II/2 (s. Anm. 16), 294. 
ist, zeigt sich darin, dass er in der Versöhnungslehre der Kirchlichen Dogmatik seine Rede von der Gemeinde in der Doppelgestalt von Israel und Kirche, über die sich „der Bogen des einen Bundes“ spannt, wieder aufgreift. Dies geschieht bezeichnenderweise bei der Erläuterung des Glaubenssatzes von der Einheit der Kirche - Credo unam ecclesiam -, wobei Barth im Blick auf das Judentum betont, die „entscheidende Frage“ sei nicht, was die Synagoge ohne Christus sein könne, sondern: „was ist die Kirche, solange ihr ein fremdes und entgegengesetztes Israel gegenübersteht?" Die Antwort könne keinesfalls in der „Judenmission“ bestehen, denn „was hat die Kirche, was die Synagoge (Röm 9,4-5) nicht auch hatte, lange vor ihr hatte [...]?“ So sei die „sog. Judenfrage“ in Wahrheit „die Christenfrage“" ${ }^{19}$

Für die zweite Vollversammlung des Ökumenischen Rats der Kirchen, die 1954 in Evanston (USA) unter dem Motto „Jesus Christus - die Hoffnung der Welt“ abgehalten wurde, hat Barth auf der Grundlage seiner „Israellehre“ einen Textvorschlag erarbeitet, der von der „Hoffnung Israels" redet, an der die Kirche in Christus partizipiere. Er schließt mit dem Satz: „Das Problem der Einheit der Kirche mit Israel ist das erste Problem der ökumenischen

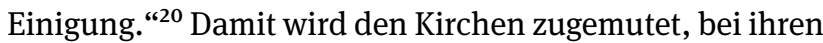
Bemühungen um „ökumenische Einigung“ ihre von Gott bereits gestiftete „Einheit“ mit Israel als „Problem“ und damit als Aufgabe zu bedenken. ${ }^{21}$ Offenbar setzt Barth auch hier den „Bogen des einen Bundes“, der sich über beide Gestalten der Gemeinde wölbt, als durch Gottes Gnadenwahl gegeben voraus. Eine Einigung der christlichen Konfessionen an Israel vorbei muss in dieser Perspektive als Selbstmissverständnis der ökumenischen Bewegung erscheinen.

Barths Textvorschlag führte auf der Vollversammlung von Evanston jedoch zu scharfen Kontroversen. Ein Passus über die „Hoffnung Israels“, der sein Anliegen aufgenommen hätte, wurde aus dem Abschlussbericht gestrichen. Daraufhin gaben 24 Delegierte der unterlegenen Minderheit eine eigene Erklärung über die „Hoffnung Israels“ ab, die allen im Ökumenischen Rat vertretenen Kirchen zugeleitet wurde. Der Generalsekretär des Ökumenischen Rates, Willem A. Visser 't Hooft, kommentierte den Streit im Nachhinein, indem er der siegreichen Mehrheit implizit Antisemitismus vorwarf: „Was steckte hinter alledem?

19 Karl Barth, Die Kirchliche Dogmatik, Bd. IV: Die Lehre von der Versöhnung. Erster Teilband, Zollikon-Zürich 1953, 749.

20 Vgl. Karl Barth, Die Hoffnung Israels; zit. nach Paul Démann, Israel in Evanston, in: Freiburger Rundbrief 8 (1955), 25-30, hier: $26 \mathrm{f}$. 21 Vgl. Matthias Loerbroks, Weisung vom Zion. Biblisch-theologische Orientierungen für eine Kirche neben Israel, Berlin 2000, 45.
Während der entscheidenden Abstimmung, während ich vom Podium aus beobachtete, wie die nationalen Delegationen stimmten, sagte ich mir: Hier geht Hitlers Geist um. ${ }^{\text {"22 }}$

Barth blieb seiner Auffassung trotz der Niederlage von Evanston treu. So hat er im dritten Teil der Versöhnungslehre der Kirchlichen Dogmatik ausdrücklich Konsequenzen für die innerchristliche Ökumene aus seiner „Israellehre“ gezogen. Dort heißt es: „Auch die ökumenische Bewegung von heute leidet schwerer unter der Abwesenheit Israels, als unter der Roms und Moskaus! [...] Die immer wieder aufbrechende Judenfrage ist die durch keinen ihrer Dienste beantwortete und zu beantwortende Christenfrage und Kirchenfrage." ${ }^{23}$ Vorausgesetzt ist auch hier eine „klare Ablehnung der christlichen Judenmission“, weil ein sich „seines Judentums bewußter und also ernstzunehmender Jude [...] sich schon mit diesem Wort nur eben mißverstanden und beleidigt finden" müsse. ${ }^{24}$

Aus Anlass seines Besuchs in Rom im Jahr 1966 hat Barth schließlich mündlich geäußert: „Die ökumenische Bewegung wird deutlich vom Geiste des Herrn getrieben. Aber wir sollten nicht vergessen, daß es schließlich nur eine tatsächlich große ökumenische Frage gibt: unsere Beziehungen zum Judentum. “25 Der katholische Theologe Hans Hermann Henrix (Aachen) hat diese Mahnung als „Karl Barths ökumenisches Testament“ gewürdigt. ${ }^{26}$ Und der katholische Fundamentaltheologe Johann Baptist Metz (Münster) hat unter Berufung auf Barths Formulierung vorsichtig seine Hoffnung auf eine künftige „Koalition des messianischen Vertrauens zwischen Juden und Christen" angedeutet. ${ }^{27}$

22 W. A. Visser 't Hooft, Die Welt war meine Gemeinde, München 1972, 300; zit. nach Loerbroks, Weisung vom Zion (s. Anm. 21), 47. Man kann fragen, ob die unklare Haltung des Ökumenischen Rates bis heute, die sich auch in Sympathiebekundungen für Kampagnen wie „Boycott, Divestment, and Sanctions“ (BDS) gegen Israel äußert, nicht als Spätfolge des damaligen Versagens in Evanston verstanden werden muss.

23 Karl Barth, Die Kirchliche Dogmatik, Bd. IV. Dritter Teilband, Zollikon-Zürich 1959, 1007.

24 Barth, Die Kirchliche Dogmatik, Bd. IV/3 (s. Anm. 23), 1005.

25 Karl Barth anlässlich seines Rombesuchs 1966 gegenüber Mitgliedern des vatikanischen Sekretariats für die Einheit der Christen; zit. nach Hans Hermann Henrix, „Ökumenische Theologie und Judentum. Gedanken zur Nichtexistenz, Notwendigkeit und Zukunft eines Dialogs“, in: Freiburger Rundbrief 28 (1976), 27.

26 Vgl. Henrix, „Ökumenische Theologie und Judentum“(s. Anm. 25), 27, Anm. 58.

27 Johann Baptist Metz, „Ökumene nach Auschwitz - Zum Verhältnis von Christen und Juden in Deutschland“, in: Eugen Kogon, Johann Baptist Metz u.a., Gott nach Auschwitz. Dimensionen des Massenmords am jüdischen Volk, Freiburg i.Br. 1979, 121-144, hier: 144. 
Wie bedeutsam dieses ökumenische Testament für Barth war, lässt sich seiner Stellungnahme zu den Beschlüssen des Zweiten Vatikanischen Konzils entnehmen. So hat er kritisch zurückgefragt, warum „das GrundSchisma - der Gegensatz von Kirche und Synagoge“ - nicht im Dekret über den Ökumenismus, wo es hingehört hätte, behandelt wird; offenbar wirkte es auf Barth deplatziert, dass „die Beziehung der Kirche zur ,Nachkommenschaft Abrahams “" erst in der Deklaration Nostra Aetate über das Verhältnis der Kirche zu den nichtchristlichen Religionen zur Sprache gebracht wird. ${ }^{28} \mathrm{Zu}$ dieser Erklärung formulierte Barth kritische Anfragen: „Wie kommt die ,Deklaration' dazu [...], im Blick auf Israels Geschichte und Gegenwart - in einem Atemzug mit Hinduismus, Buddhismus, Moslemismus - von einer ,nicht-christlichen Religion' zu reden, wo es sich doch a) im Alten Testament keineswegs um eine ,Religion', sondern um die Urgestalt der einen Gottesoffenbarung[,] b) in der Existenz des späteren und heutigen (gläubigen oder ungläubigen) Judentums um den einen einzigen natürlichen (weltgeschichtlichen) Gottesbeweis handelt?" 29

Schließlich vermisste Barth in Nostra Aetate die Anerkennung einer kirchlichen Schuld gegenüber den Juden: „Wäre hier nicht [...] angesichts der judenfeindlichen Haltung der alten, der mittelalterlichen und weithin auch der modernen Kirche ein ausdrückliches Schuldbekenntnis am Platz gewesen?“ Und Barth weitete die Perspektive des Schuldbekenntnisses abschließend auf das christlichmuslimische Verhältnis aus, indem er fragte, ob nicht „bei Erwähnung der Muslim[e] ein solches in Erinnerung an die fatale Rolle der Kirche in den sogenannten Kreuzzügen“ am Platz gewesen wäre. ${ }^{30}$ In diesen Äußerungen des alten Karl Barth deutet sich bereits so etwas wie eine „abrahamitische Ökumene“ zwischen Juden, Christen und Muslimen an, von der heute gelegentlich die Rede ist. ${ }^{31}$

28 Karl Barth, Ad Limina Apostolorum, Zürich 1967, 33.

29 Barth, Ad Limina (s. Anm. 28), $39 \mathrm{f}$.

30 Barth, ebd.

31 Schon im Jahr 1956 hatte der liberale Rabbiner Leo Baeck von Juden, Christen und Muslimen unter dem Aspekt dessen gesprochen, was sie trennt und was sie eint. Vgl. Leo Baeck, „Judentum, Christentum und Islam. Was sie trennt, was sie eint“ (1956), in: ders., Nach der Schoa - Warum sind die Juden in der Welt? Schriften aus der Nachkriegszeit, hg. v. Albert H. Friedlander u. Bertold Klappert (= Leo Baeck Werke, Bd. 5), Gütersloh 2006, 472-489.

\section{Folgerungen und Ausblick}

Würde man Karl Barths Rede vom christlich-jüdischen Verhältnis als der „einen tatsächlich großen ökumenischen Frage“ ernst nehmen, müsste sich der Begriff von Ökumene ändern. Die ökumenische Bewegung könnte ihr Selbstverständnis nicht mehr exklusiv vom Christusbekenntnis abhängig machen. Sie müsste sich stattdessen als eine Gemeinschaft bleibend Verschiedener verstehen, die nicht auf dem gemeinsamen Bekenntnis, sondern auf der Anerkennung der Alterität des Anderen beruhen würde.

Damit würde aber auch die Rede von der ökumenischen „Einheit in versöhnter Verschiedenheit“ in ein neues Licht treten. Denn die Einheitskriterien der innerchristlichen Ökumene wie etwa die Gemeinsamkeit in der einen Taufe, in der einen Eucharistie und im geistlichen Amt, die zu einer „völlig verpflichtenden Gemeinschaft“ führen sollen, können zwischen Juden und Christen keine Geltung beanspruchen. Zwar haben Juden und Christen religionsgeschichtlich gemeinsame Wurzeln; dies bedeutet aber durchaus nicht, dass sie auch theologisch auf eine übergreifende „Einheit“ hin angelegt wären. Das Auseinandergehen der Wege kann und soll nicht rückgängig gemacht werden. Das hat die im Dezember 2015 veröffentlichte Erklärung orthodoxer Rabbiner Den Willen unseres Vaters im Himmel tun: Hin zu einer Partnerschaft zwischen Juden und Christen in eindrücklicher Weise betont, die zu den Veränderungen im Verhältnis von Christen und Juden in den letzten Jahrzehnten seit Nostra Aetate Stellung nimmt. ${ }^{32}$ Und doch legt sich aufgrund der gemeinsamen Wurzel die Frage nahe, ob und inwiefern Juden und Christen auch heute theologisch in ein geschwisterliches Verhältnis zueinander treten können und sollen.

Was müsste sich im Selbstverständnis und in der Struktur der ökumenischen Bewegung ändern, damit Juden sich beteiligen können, ohne sich christlich vereinnahmt fühlen zu müssen? Können hier die Gesellschaften für christlich-jüdische Zusammenarbeit als Modell dienen? Oder müsste auch deren Selbstverständnis und Struktur im Blick auf ein Neues überwunden werden? Müsste nicht auch in der engeren Ökumene zwischen den christlichen Kirchen die Anerkennung der bleibenden Verschiedenheit der Konfessionen als der einzig gangbare Weg zur Versöhnung gelten? Sollte hier nicht eher von dialogischer Pluralität als von Einheit die Rede sein?

32 Der Wortlaut ist $\mathrm{zu}$ finden unter www.jcrelations.net/de/ statements/statement/den-willen-unseres-vaters-im-himmel-tunhin-zu-einer-partnerschaft-zwischen-juden-und-christen.html. 
Bezeichnenderweise wurde ja der Konflikt Martin Luthers mit der Römischen Kirche im 16. Jahrhundert von jüdischer Seite zunächst mit einigen Hoffnungen begleitet. Der Zusammenstoß zwischen Protestanten und Katholiken wurde als Bruch innerhalb der monolithischen Macht der Kirche begrüßt. Manche hofften gar, dass die Unruhe, die innerhalb der christlichen Welt durch die Verbreitung des Luthertums entstanden war, zu einer Tolerierung aller abweichenden Glaubensweisen führen werde. ${ }^{33}$ Demgegenüber besteht bei innerchristlichen ökumenischen Einigungen ohne Rücksicht auf das Judentum die Gefahr eines Konsenses auf Kosten Dritter. So hat Hans Hermann Henrix schon 1976 vor einer „unbedachten innerchristlichen Konsensbildung zu Lasten des Judentums “ gewarnt. ${ }^{34}$ Und Friedrich-Wilhelm Marquardt hat aus Anlass der „Gemeinsamen Erklärung zur Rechtfertigungslehre“, die 1999 vom Lutherischen Weltbund und vom Päpstlichen Rat zur Förderung der Einheit der Christen verabschiedet worden war, bedauert, dass sich hier Protestanten und Katholiken „konsequent am Judentum vorbei“ geeinigt hätten, so dass gesagt werden müsse: „Im Antijudaismus einigen sich die christlichen Kirchen.“"35

Wenn man die Formel von der „versöhnten Verschiedenheit" auf das christlich-jüdische Verhältnis anwenden wollte, dann müsste sie so verstanden werden, dass die Versöhnung nur unter Voraussetzung der Anerkennung bleibender Verschiedenheit möglich wird. Das Ziel wäre nicht „Einheit“, sondern eine „Ökumene“ zwischen Juden und Christen, die sich dialogisch als ein Weg der Versöhnung ohne Angst vor Vereinnahmung des Anderen verwirklicht. So könnten die Verschiedenen ohne Angst voreinander verschieden bleiben und sich doch zugleich als eine Weg-Gemeinschaft verstehen lernen.

Der Apostel Paulus hat in seinem Brief an die Römer von einem edlen Wettstreit zwischen Juden und Nichtjuden im Blick auf die Treue zum Gott Israels geredet: Das ihm unverständliche Nein der jüdischen Mehrheit zur Messianität Jesu diene wohl dazu, dass die messianische Botschaft zu den Nichtjuden komme, um auf diesem Umweg wiederum die Juden „eifersüchtig“ zu machen (Röm 11,11). In diesem Zusammenhang redet er von einem „Geheim-

33 Vgl. Andreas Pangritz, „Zeitgenössische jüdische Reaktionen auf Luther und die Wittenberger Reformation“, in: Begegnungen. Zeitschrift für Kirche und Judentum 94 (2011), H. 1, 2-9.

34 Henrix, „Ökumenische Theologie und Judentum“ (s. Anm. 25), 26. 35 Friedrich-Wilhelm Marquardt, „Vom Rechtfertigungsgeschehen zu einer Evangelischen Halacha“, in: Hans Martin Dober u. Dagmar Mensink (Hg.), Die Lehre von der Rechtfertigung des Gottlosen im kulturellen Kontext der Gegenwart. Beiträge im Horizont des christlich-jüdischen Gesprächs, Stuttgart 2002 (Hohenheimer Protokolle; Bd. 57), 43-75, hier: $43 \mathrm{f}$. nis“, das darin bestehe, dass die Juden zwar „nach dem Evangelium [...] Feinde um euretwillen“ geworden seien, nach der göttlichen „Erwählung“ aber „Geliebte um der Väter willen“ (Röm 11,28) blieben.

Karl Barth hat aus den Erwägungen des Apostels Paulus über eine Ökumene des Wettstreits zwischen Juden und Christen die Konsequenz gezogen, von der „einen Gemeinde Gottes“ in der Doppelgestalt von Israel und der Kirche unter dem „Bogen des einen Bundes“ zu reden. Aber vielleicht ist auch in dieser Formel der Aspekt der Einheit angesichts der tatsächlichen Trennung der Wege noch überbetont. Vielleicht ist hier die Gefahr der christlichen Vereinnahmung des Judentums noch nicht scharf genug gesehen - trotz Barths ausdrücklicher Ablehnung der Judenmission. Daher hat der Barth-Schüler FriedrichWilhelm Marquardt betont: „Wir werden den christlichen Antijudaismus erst hinter uns haben, wenn es uns theologisch gelingt, mit dem jüdischen Nein zu Jesus Christus etwas Positives anzufangen. “36 Denn ,im jüdischen Selbstverständnis“ sei ja gerade „das Nein zu Jesus Christus ein Akt der Treue zur Tora““. ${ }^{37}$ Gott wisse nun einmal „,viel tausend Weisen [...], zu retten aus der Not“, und „mindestens zwei davon“ könnten wir „in ihrer Unterschiedlichkeit und Verschiedenheit theologisch klar erkennen“. Gott sei „anders den Juden, anders den Nichtjuden Gott“.38

Aufgrund der dargestellten Probleme würde ich dafür plädieren, die Rede von der „Einheit in versöhnter Verschiedenheit“ durch eine „versöhnte Verschiedenheit in dialogischer Differenz" ${ }^{\text {“39 }}$ als Modell ökumenischer Verständigung zu ersetzen. Auch und gerade im Blick auf die Begegnung von Christen und Juden wäre eine dialogische „Weggemeinschaft“ in versöhnter Verschiedenheit anzustreben. ${ }^{40}$ Die zentrale ökumenische Frage würde dann nicht mehr lauten, wie die christlichen Konfessionen sich einigen können, auch nicht, ob Juden sich zu Jesus als dem Christus bekennen können, sondern ob die Christen

36 Friedrich-Wilhelm Marquardt, „,Feinde um unsretwillen“. Das jüdische Nein und die christliche Theologie“ (1977), in: ders, Verwegenheiten. Theologische Stücke aus Berlin, München 1981, 311-336, hier: 311.

37 Marquardt, „Feinde um unsretwillen“(s. Anm. 36), 315.

38 Marquardt, „Feinde um unsretwillen“ (s. Anm. 36), 326.

39 Christoph Schwöbel, „Ökumenische Verständigung. Versöhnte Verschiedenheit in dialogischer Differenz", in: Das Ende der Toleranz? Identität und Pluralismus in der modernen Gesellschaft, hg. v. d. Alfred Herrhausen Gesellschaft für internationalen Dialog, München / Zürich 2002, 169-175.

40 Vgl. Daniela Koeppler, Predigen aus der Hebräischen Bibel. Mit Impulsen aus jüdischer Tradition, hg. v. d. Evangelisch-Lutherischen Landeskirche Hannovers, Hannover 2019, 29. 
bereit sind, sich zur Bundesgeschichte Gottes mit seinem Volk Israel zu bekennen.

So käme die Vorstellung von der Einheit der Menschheit in bleibender Verschiedenheit zur Geltung, wie jüdische Tradition sie in der Mischna formuliert: „Der Mensch wurde deshalb einzig erschaffen [...], um die Größe des Heiligen, gepriesen sei er, zu verkünden; wenn ein Mensch mehrere Münzen mit einem Stempel prägt, so gleichen sie alle einander, der König der Könige aber, der Heilige, gepriesen sei er, prägt jeden Menschen mit dem Stempel des Urmenschen, und doch gleicht nicht einer dem anderen.“41
Wenn das Jahr der Erinnerung an 1700 Jahre jüdisches Leben in Deutschland die Kirchen ermutigen würde, in diesem Sinn von jüdischer Tradition zu lernen, wäre es nicht umsonst.

\section{Autoreninformationen}

Prof. em. Dr. Andreas Pangritz, zuletzt Lehrstuhl für Systematische Theologie an der Evangelisch-Theologischen Fakultät der Universität Bonn und zugleich einer der beiden Direktoren des Ökumenischen Instituts

41 Mischna Sanhedrin IV,5; zit. nach Der Babylonische Talmud, nach der ersten zensurfreien Ausgabe unter Berücksichtigung der neueren Ausgaben und handschriftlichen Materials ins Deutsche übersetzt von Lazarus Goldschmidt, Frankfurt am Main 1996, Bd. VIII. 